\title{
The interpretation of non-Markovian stochastic Schrödinger equations as a hidden-variable theory
}

\author{
Jay Gambetta and H. M. Wiseman* \\ Centre for Quantum Dynamics, School of Science, Griffith University, Brisbane 4111, Australia
}

(Dated: 8 July 2003)

\begin{abstract}
Do diffusive non-Markovian stochastic Schrödinger equations (SSEs) for open quantum systems have a physical interpretation? In a recent paper [Phys. Rev. A 66, 012108 (2002)] we investigated this question using the orthodox interpretation of quantum mechanics. We found that the solution of a non-Markovian SSE represents the state the system would be in at that time if a measurement was performed on the environment at that time, and yielded a particular result. However, the linking of solutions at different times to make a trajectory is, we concluded, a fiction. In this paper we investigate this question using the modal (hidden variable) interpretation of quantum mechanics. We find that the noise function $z(t)$ appearing in the non-Markovian SSE can be interpreted as a hidden variable for the environment. That is, some chosen property (beable) of the environment has a definite value $z(t)$ even in the absence of measurement on the environment. The non-Markovian SSE gives the evolution of the state of the system "conditioned" on this environment hidden variable. We present the theory for diffusive non-Markovian SSEs that have as their Markovian limit SSEs corresponding to homodyne and heterodyne detection, as well as one which has no Markovian limit.
\end{abstract}

PACS numbers: 03.65.Ta, 03.65.Yz, 42.50.Lc

\section{INTRODUCTION}

In nature it is very unlikely to find a system existing in isolation; usually it is immersed in an environment (or bath). In quantum mechanics we label this type of system an open quantum system [1]. To determine the evolution we must solve the Schrödinger equation

$$
d_{t}|\Psi(t)\rangle=-\frac{i}{\hbar} \hat{H}_{\mathrm{uni}}(t)|\Psi(t)\rangle
$$

where $|\Psi(t)\rangle$ and $\hat{H}_{\text {uni }}(t)$ are the quantum state and Hamiltonian for the complete universe (system and bath). That is, $|\Psi(t)\rangle$ belongs to the Hilbert space $\mathcal{H}_{\text {uni }}=\mathcal{H}_{\text {sys }} \otimes \mathcal{H}_{\text {bath. }}$. Due to the large Hilbert space of the bath $\left(\mathcal{H}_{\text {bath }}\right)$ it is convenient to describe the system by its reduced state. This is defined as

$$
\rho_{\text {red }}(t)=\operatorname{Tr}_{\text {bath }}[|\Psi(t)\rangle\langle\Psi(t)|],
$$

and operates only in the $\mathcal{H}_{\text {sys }}$.

It has been shown $[2,3]$ by a projection-operator method that we can write a general master equation for the reduced state as

$$
d_{t} \rho_{\text {red }}(t)=-\frac{i}{\hbar}\left[\hat{H}(t), \rho_{\text {red }}(t)\right]+\int_{0}^{t} \hat{\mathcal{K}}(t, s) \rho_{\text {red }}(s) d s,
$$

where $\hat{H}(t)$ operates only in $\mathcal{H}_{\text {sys }}$ and $\hat{\mathcal{K}}(t, s)$ is the "memory time" superoperator. It operates on $\rho_{\text {red }}(s)$ and represents how the bath has affected the system in the past. The problem with this equation is that in general $\hat{\mathcal{K}}(t, s)$ cannot be explicitly evaluated.

\footnotetext{
*Electronic address: h.wiseman@griffith.edu.au
}

In recent papers $[4,5,6,7,8,9,10,11,12,13]$ nonMarkovian stochastic Schrödinger equations (SSEs) have been proposed which allow an alternative procedure for solving the reduced state. A non-Markovian SSE is a stochastic equation for the system state $\left|\psi_{z}(t)\right\rangle$, conditioned on some noise function $z(t)$. We consider only continuous SSEs, although discontinuous ones have also been proposed [14]. The SSE has the property that when the projector for $\left|\psi_{z}(t)\right\rangle$ is averaged over all the possible $z(t)$ one obtains $\rho_{\text {red }}(\mathrm{t})$. That is,

$$
\rho_{\text {red }}(t)=\mathrm{E}\left[\left|\psi_{z}(t)\right\rangle\left\langle\psi_{z}(t)\right|\right]
$$

where $\mathrm{E}[\ldots]$ denotes an ensemble average over all possible $z(t)$ 's.

When using non-Markovian SSEs to solve the reduced state it turns out that in general we cannot explicitly evaluate $\left|\psi_{z}(t)\right\rangle$. However, as shown in Refs. [15, 16] we can use perturbative techniques to find approximate solutions. Here we are interested not in how to solve the non-Markovian SSEs, but in how to interpret them.

When Markovian SSEs (the Markovian limit of nonMarkovian SSEs) were introduced in quantum optics their first interpretation was as a numerical tool [17]. Another interpretation was that Markovian SSEs represent objective (that is, independent of any observer) trajectories for the system $[18,19,20]$. In this interpretation the SSE is seen as a (stochastic and nonlinear) modification of the Schrödinger equation, modelling how state reduction (collapse of the wave function) occurs in open quantum systems. However, in recent times it has been generally accepted that Markovian SSEs are evolution equations for the system state conditioned on continuous measurement of the bath $[1,21,22,23]$.

A Markovian bath is one for which the bath correlation time is much less than the decoherence time of the system. For such a system one can envisage mak- 
ing repeated measurements on the bath on a time scale infinitesimal compared to the system decoherence time but large compared to the bath correlation time. Thus these measurements do not lead to any back-action of the system from the bath. But the measurement of the bath does yield information about the system. This can be described as the action of a measurement operator (as appears in generalized quantum measurement theory [24]) for an infinitesimal time interval [23], and keeps the system in a pure state. It can be reexpressed as a SSE in which the stochastic variable $z(t)$ is related to the results of the continuous measurements. The stochastic trajectory of this conditioned system state generated by the Markovian SSE is often referred to as a quantum trajectory [1]. Different detection procedures (measurements on the bath) result in different unravelings. A few common examples are direct detection [1], homodyne [1, 21], and heterodyne detection [22].

In the light of the quantum trajectory interpretation of Markovian SSEs, we return to the interpretation of non-Markovian SSEs. Obviously one interpretation is that they are simply a numerical tool used to generate the reduced state. However, after the success in finding a physical interpretation for Markovian SSEs, it is natural to seek something beyond this trivial interpretation. Moreover, we have previously shown [10] that there are different non-Markovian unravelings, and that these correspond to different measurement schemes (homodyne and heterodyne) in the Markovian limit. Thus it is natural to seek an interpretation of non-Markovian SSEs beyond that of being just numerical tools [25].

In Ref. [10] we came to the conclusion that under the orthodox interpretation [24] of quantum mechanics, the solution of a non-Markovian SSE at time $t$ is the state the system will be in, if at that time a measurement was performed on the bath and yielded a result $z$. Thus the non-Markovian SSE under this view has no interpretation; it is just a stencil used to calculate the conditioned system state at a particular time $t$. In other words, the linking of $\left|\psi_{z}(t)\right\rangle$ (or $z(t)$ ) with itself at times less then $t$ turns out to be a convenient fiction.

Unlike the Markovian case, it is not possible to derive the SSE by continuously measuring the bath because a non-Markovian bath has a non-negligible correlation time. Thus if a measurement is made at time $t$, collapsing the bath state at that time, this will affect the state of the bath interacting with the system in the future. That is, the measurement on the bath will cause a backaction on the system and hence the average evolution for a system state conditioned on continuous measurement of the bath will not be that of Eq. (1.2). In fact, a continuous measurement on a bath with a nonzero correlation time will lead to a quantum Zeno effect, radically altering the average evolution of the system. If, on the other hand, the measurement on the bath is not done continuously, then the system state will not remain in a pure state as the system and bath will become entangled in the time between measurements on the bath. Thus it seems safe to conclude that there is no continuous measurement interpretation, and in fact we are forced to accept that SSEs are only a numerical tool which could be used to determine the system state at a particular time conditioned on a particular measurement result $z$.

Since orthodox quantum mechanics fails to give a satisfying interpretation for non-Markovian SSEs, in this paper we turn to a nonorthodox approach: the modal interpretation of quantum mechanics $[26,27,28,29,30,31$, 32]. This interpretation, unlike the orthodox interpretation, has as its basic goal to keep reality intact. That is, the values of some observables (the hidden variables) really exist before we measure them. Because the observables have an objective reality from now on we will refer to them as properties or beables (after Bell [26]). Just as in the orthodox theory, where it is impossible to simultaneously measure all observables, in the modal theory it is impossible to give all observables property status The best-known example of such an interpretation is Bohmian mechanics for particles [33] in which position is the preferred observable (property).

We expect a modal interpretation to be applicable to non-Markovian SSEs because we can use it to assign definite properties to the bath, as occurs in the orthodox theory when the bath is measured, without invoking such a measurement. In this way we avoid the backaction problem which arose in the orthodox theory. While the bath is ascribed definite properties, the system is described as a purely quantum system. But, because of the entanglement between the system and the bath, we can define a system state associated with (or "conditioned" on) a particular value for the bath property. If the bath properties are described by rank-one projective measures on $\mathcal{H}_{\text {bath }}$, then the conditioned system state will be pure. Averaging over the conditioned system state would reproduce the non-Markovian reduced state matrix $\rho_{\text {red }}(t)$, just as in Eq. (1.4).

Since the value of the bath hidden variables change in time, the conditioned system state will evolve in time also, and this time there is a meaningful relation between the conditioned system state at different times. A particular set of bath properties amounts to a particular decomposition (of the unit operator on $\mathcal{H}_{\text {bath }}$ ), and leads to a particular unraveling of the non-Markovian master equation (1.3). We will use these terms interchangeably.

In this paper we show that for a suitable choice of bath properties we can reproduce all of the non-Markovian SSEs discussed above, as well as one which has not been previously considered. The noise function $z(t)$ appearing in the non-Markovian SSE is simply a function of the values of the bath hidden variables. The system state $\left|\psi_{z}(t)\right\rangle$ is the system state conditioned on the bath properties having the values giving $z(t)$. Moreover, the system state guides the values of the hidden variables of the bath.

The format of this paper is as follows: In Sec. II a summary of the modal interpretation of quantum mechanics is presented. In Sec. III we give the microscopic 
model for non-Markovian dynamics for an open quantum system that underlies all of our work. In Sec. IV we show how modal dynamics can be applied to such models, and derive the hidden variable interpretation of nonMarkovian SSEs for three different unravelings. These correspond to objective values for the position of each bath harmonic oscillators (as in the Bohmian interpretation), for the quadrature of the collective bath field, and for the coherent amplitude of the collective bath field. The first of these has no Markovian limit, while the latter two have homodyne and heterodyne detection as their Markovian limit. Lastly in Sec. V we conclude with a discussion and directions for future work.

\section{MODAL INTERPRETATION OF QUANTUM MECHANICS}

In this section we give a brief overview of the modal interpretation of quantum mechanics; for a more detailed description see Refs. $[26,27,28,29,30,31,32]$. The basic idea of this view of quantum mechanics is that certain observables have a definite reality independent of measurement, whereas in the orthodox interpretation the measurement defines the reality of the observable. To explain this we consider measurement of the observable $Z$. The notation we use to represent an observable is

$$
Z=\left\{\left(z_{n}, \hat{\pi}_{n}\right)\right\} .
$$

That is, $Z$ is represented by a set of paired elements. Each pair represents the value $z_{n}$ and the corresponding projector $\hat{\pi}_{n}$. The projectors are orthogonal and form a decomposition of unity:

$$
\sum_{n} \hat{\pi}_{n}=\hat{1}
$$

In Eq. (2.1) for simplicity we have only considered the case where $Z$ is time independent. We can in this case also define an operator $\hat{Z}$ which is equivalent to Eq. (2.1), by

$$
\hat{Z}=\sum_{n} z_{n} \hat{\pi}_{n}
$$

In the orthodox interpretation $Z$ has a definite value $z_{n}$ if and only if $|\Psi(t)\rangle=\left|\Psi_{n}(t)\right\rangle$ (an eigenstate of $\hat{Z}$ ). However, in general $|\Psi(t)\rangle=\sum_{n} c_{n}(t)\left|\Psi_{n}(t)\right\rangle$, which implies that $Z$ has all values contained in the sum; it is not well defined. Upon measurement, by the introduction of a reduction equation $\left[|\Psi(t)\rangle \rightarrow\left|\Psi_{n}(t)\right\rangle\right]$ the value of $Z$ becomes $z_{n}$ with probability $\left|c_{n}\right|^{2}$.

In the modal interpretation we choose one projective measure, $\left\{\hat{\pi}_{n}\right\}$, as the preferred measure. This then determines which observables can be given property status. To explain why the property takes the value $z_{n}$ we introduce an extra quantum state, the property state,

$$
\left|\Psi_{n}(t)\right\rangle=\hat{\pi}_{n}|\Psi(t)\rangle / \sqrt{N}
$$

where $N$ is a normalization constant. This state propagates in time along with $|\Psi(t)\rangle$, except it is stochastic in nature (jumps between different $n$ ). It is interpreted as the actual state of the universe, by the eigenstate eigenvalue it has a definite value $\left(z_{n}\right)$ for $Z$. The stochastic dynamics (rates at which it jumps between different $n$ ) is determined by $|\Psi(t)\rangle$ and so in this interpretation $|\Psi(t)\rangle$ is called the guiding state.

For the purposes of this paper Eq. (2.3) is actually not general enough. In Ref. [32] we showed that this theory can be extended to positive operator measures (POMs) [34], that is

$$
Z=\left\{\left(z_{n}, \hat{F}_{n}\right)\right\}
$$

where $\hat{F}_{n}$ is an effect (or POM element) with $\sum_{n} \hat{F}_{n}=\hat{1}$. In Eq. (2.5), $z_{n}$ is the value of the effect, which could be a real number, a complex number, a string of numbers, or even a statement (yes/no).

In Ref. [32] we showed that by implementing Naimark's theorem, modal dynamics can be extended to include POMs. Naimark's theorem says that if we enlarge the Hilbert space of the universe from $\mathcal{H}_{\text {uni }}$ to $\mathcal{K}=\mathcal{H}_{\text {uni }} \otimes \mathcal{H}_{\text {aux }}$, we can define a projector $\hat{\Pi}_{n}$ in $\mathcal{K}$ such that

$$
\left\langle\Psi(t)\left|\hat{F}_{n}\right| \Psi(t)\right\rangle=\left\langle\Psi(t)\left|\left\langle\phi\left|\hat{\Pi}_{n}\right| \phi\right\rangle\right| \Psi(t)\right\rangle,
$$

for all $|\Psi(t)\rangle \in \mathcal{H}_{\text {uni }}$ and for all possible $n .|\phi\rangle\langle\phi|$ is called the Naimark projection of $\mathcal{K}$ onto $\mathcal{H}_{\text {uni }}$. To work out the set $\left\{\hat{\Pi}_{n}(t)\right\}$ it is necessary to introduce another projector $\hat{\Pi}_{N+1}(t)$, such that

$$
\sum_{n}^{N+1} \hat{\Pi}_{n}(t)=\hat{1}_{\text {uni }+ \text { aux }}
$$

and

$$
\hat{\Pi}_{n}(t) \hat{\Pi}_{m}(t)=\hat{\Pi}_{n}(t) \delta_{n m}
$$

is satisfied for $n, m,=1, \ldots, N+1$. The set of projectors in this enlarged Hilbert space is called the Naimark extension of $\hat{F}_{n}(t)$ [34]. Worked examples of this are shown in [34]. In this enlarged space the observable defined by Eq. (2.5) becomes the property

$$
Z=\left\{\left(z_{n}, \hat{\Pi}_{n}\right)\right\},
$$

or equivalently

$$
\hat{Z}=\sum_{n}^{N+1} z_{n} \hat{\Pi}_{n} .
$$

Here $\left\{\hat{\Pi}_{n}\right\}$ is the preferred projective measure in $\mathcal{K}$. The guiding state becomes

$$
|\Phi(t)\rangle=|\Psi(t)\rangle \otimes|\phi\rangle,
$$


where $|\Psi(t)\rangle$ is still the solution to the Schrödinger equation (1.1). The property state becomes

$$
\left|\Phi_{n}(t)\right\rangle=\hat{\Pi}_{n}|\Phi(t)\rangle / \sqrt{N} .
$$

That is, when describing POMs the universe is described by a property state in the enlarged Hilbert space, which does not factorize into a universe state and an auxiliary state. This can be regarded merely as a mathematical construction to give reality to POMs. Note that the value of $z_{N+1}$ is irrelevant as $\hat{\Pi}_{N+1}$ projects into the null space of $|\Phi(t)\rangle$. That is this value will occur with zero probability.

The modal dynamics [the stochastic evolution of the states in Eq. (2.4) or Eq. (2.12)] is found using the method originally proposed by Bell [26] and generalized in Refs. [29, 30, 32] to include time dependent projectors and POMs. Define $P_{n}(t)$ as the probability that the system is in the $n^{\text {th }}$ state at time $t$. Assuming a Markovian process, by which we mean that the probability of being in state $m$ at time $t+d t$ only depends on the state at time $t$, we can write a master equation for $P_{n}(t)$ as

$$
d_{t} P_{n}(t)=\sum_{m}\left[T_{n m}(t) P_{m}(t)-T_{m n} P_{n}(t)\right],
$$

where $T_{n m}$ are transition rates. For $n=m, T_{n n}$ (which is negative) is a measure of the rate at which state $n$ losses probability.

Defining a probability current $J_{n m}(t)$ as

$$
J_{n m}(t)=T_{n m}(t) P_{m}(t)-T_{m n} P_{n}(t)
$$

results in $J_{n m}(t)=-J_{m n}(t)$ and allows us to rewrite the probability master equation as

$$
d_{t} P_{n}(t)=\sum_{m} J_{n m}(t)
$$

Given $J_{n m}(t)$ and $P_{n}(t)$, there are many possible transition rates satisfying Eq. (2.15). One solution, chosen by Bell [26] is as follows.

For $J_{n m}(t)<0$,

$$
\begin{aligned}
& T_{n m}(t)=0 \\
& T_{m n}(t)=-J_{n m}(t) / P_{n}(t),
\end{aligned}
$$

and for $J_{n m}(t)>0$

$$
\begin{aligned}
& T_{n m}(t)=J_{n m}(t) / P_{m}(t), \\
& T_{m n}(t)=0 .
\end{aligned}
$$

This is only one of the infinitely many solutions. These are found by adding an extra term $T_{n m}^{0}(t)$ to $T_{n m}(t)$, where $T_{n m}^{0}(t)$ is constrained only by

$$
T_{n m}^{0}(t) P_{m}(t)-T_{m n}^{0}(t) P_{n}(t)=0 .
$$

In Ref. [32] we showed that one possible solution for $J_{n m}(t)$ is

$$
\begin{aligned}
J_{n m}(t)= & 2 \operatorname{Im}\left\{\langle\Phi(t)| \hat{\Pi}_{n}\left[\hat{H}_{\text {uni }}(t) \otimes \hat{1}_{\text {aux }}\right]\right. \\
& \left.\times \hat{\Pi}_{m}|\Phi(t)\rangle\right\} / \hbar
\end{aligned}
$$

Note that this is only one of infinitely many possible currents, as we can add any current $J_{n m}^{0}(t)$ to $J_{n m}(t)$ which satisfies $\sum_{m} J_{n m}^{0}=0$, to give a valid probability current. For the purposes of this paper we only consider the simple solutions [not containing the extra $T_{n m}^{0}(t)$ and $J_{n m}^{0}(t)$ terms].

The above dynamics only describe a discrete decomposition. For non-Markovian SSEs we must consider continuous decompositions. In Ref. [32] we showed that provided the Hamiltonian is at most quadratic in the conjugate variable to the chosen hidden variable, then the above dynamics reduces to a deterministic theory. The only stochasticity is due to the random initial conditions of the hidden variable. That is, it is similar to Bohmian mechanics [33]. In fact if the preferred projective measure is chosen to be the position projective measure $\left(\hat{\pi}_{x}=|x\rangle\langle x|\right)$ then Bohmian mechanics is the continuum limit.

We define continuous decompositions by the preferred projective measure $\left\{\hat{\Pi}_{\left\{q_{k}\right\}}=\left|\left\{q_{k}\right\}\right\rangle\left\langle\left\{q_{k}\right\}\right|\right\}$, which in turn defines $\left\{\hat{q}_{k}\right\}$ as the operators for the hidden variables. Here we have introduced a notation for the set of hidden variables $\left(\left\{q_{k}\right\}\right)$ whose relevance will become important in the following sections. The decomposition of unity is

$$
\int\left|\left\{q_{k}\right\}\right\rangle\left\langle\left\{q_{k}\right\}\right| \prod_{k} d q_{k}=\hat{1}
$$

For this set of hidden variables we will see later that it is also useful to define a single property $Z(t)$ as

$$
Z(t)=\left\{\left(z\left(\left\{q_{k}\right\}, t\right),\left|\left\{q_{k}\right\}\right\rangle\left\langle\left\{q_{k}\right\}\right|\right)\right\},
$$

where $z\left(\left\{q_{k}\right\}, t\right)$ is a function of the values of the hidden variables $\left\{q_{k}\right\}$ and $t$.

As in Bohmian mechanics the evolution of each value of the hidden hidden variable $q_{k}(t)$ (or the corresponding property state) can be found by the differential equation

$$
d_{t} q_{k}(t)=\left.v_{k}\left(\left\{q_{j}\right\}, t\right)\right|_{q_{k}=q_{k}(t)},
$$

where $v_{k}\left(\left\{q_{j}\right\}, t\right)$ is the velocity field. This is defined as [32]

$$
v_{k}\left(\left\{q_{j}\right\}, t\right)=\frac{\operatorname{Re}\left[\left\langle\Phi(t) \mid\left\{q_{j}\right\}\right\rangle\left\langle\left\{q_{j}\right\}\left|\hat{v}_{k}(t)\right| \Phi(t)\right\rangle\right]}{\left\langle\Phi(t) \mid\left\{q_{j}\right\}\right\rangle\left\langle\left\{q_{j}\right\} \mid \Phi(t)\right\rangle} .
$$

Here $\hat{v}_{k}(t)$ is the $k^{\text {th }}$ component of the velocity operator and is defined as

$$
\hat{v}_{k}(t)=-\frac{i}{\hbar}\left[\hat{q}_{k}, \hat{H}_{\mathrm{uni}}(t) \otimes \hat{1}_{\mathrm{aux}}\right] .
$$

\section{NON-MARKOVIAN DYNAMICS FOR OPEN QUANTUM SYSTEMS}

The aim of this section is to outline the underlying dynamics used to generate non-Markovian SSEs. Firstly we assume that there are only two systems, a system 
of interest and a bath. That is, the Hamiltonian of the universe is

$$
\hat{H}_{\text {sys }} \otimes \hat{1}+\hat{1} \otimes \hat{H}_{\text {bath }}+\hat{V} \text {. }
$$

The system Hamiltonian $\hat{H}_{\text {sys }}$ is split into two terms, these being $\hat{H}_{\Omega}$ and $\hat{H}$. The bath is modelled by a collection of one-dimensional harmonic oscillators. In terms of the bath annihilation and creation operators, $\hat{a}_{k}$ and $\hat{a}_{k}^{\dagger}$, the Hamiltonian for the bath is

$$
\hat{H}_{\mathrm{bath}}=\sum_{k} \hbar \omega_{k} \hat{a}_{k}^{\dagger} \hat{a}_{k}
$$

The interaction Hamiltonian $\hat{V}$ we assume is linear in the bath amplitude, and has the form

$$
\hat{V}=i \hbar \sum_{k}\left[\hat{L} g_{k}^{*} \hat{a}_{k}^{\dagger}-\hat{L}^{\dagger} g_{k} \hat{a}_{k}\right]
$$

where $\hat{L}$ is the system lowering operator and $g_{k}$ is the coupling strength of the $k^{\text {th }}$ mode to the system. Without loss of generality we can take the $g_{k}$ 's to be real, absorbing any phases in the definitions of the bath operators.

For calculation purposes we define an interaction frame such that the fast dynamics placed on the state by the Hamiltonians $\hat{H}_{\Omega}$ and $\hat{H}_{\text {bath }}$ is moved to the operators. The unitary evolution operator for this transformations is

$$
\hat{U}_{0}(t, 0)=e^{-i\left(\hat{H}_{\Omega} \otimes \hat{1}+\hat{1} \otimes \hat{H}_{\text {bath }}\right)(t-0) / \hbar} .
$$

Thus the combined state in the interaction frame is define as

$$
|\Psi(t)\rangle=\hat{U}_{0}^{\dagger}(t, 0)\left|\Psi(t)_{\mathrm{Sch}}\right\rangle,
$$

and an arbitrary operator $\hat{A}$ becomes

$$
\hat{A}_{\text {int }}(t)=\hat{U}_{0}^{\dagger}(t, 0) \hat{A} \hat{U}_{0}(t, 0) .
$$

This allows us to write the Schrödinger equation as

$$
d_{t}|\Psi(t)\rangle=-\frac{i}{\hbar} \hat{H}_{\mathrm{uni}}(t)|\Psi(t)\rangle,
$$

where $\hat{H}_{\text {uni }}(t)=\hat{H}_{\text {int }}(t)+\hat{V}_{\text {int }}(t)$. Here $\hat{H}_{\text {int }}(t)$ refers to $\hat{H}(t)$ in the interaction picture and the form of the latter is

$$
\hat{V}_{\mathrm{int}}(t)=i \hbar \sum_{k}\left[\hat{L} g_{k} e^{i \Omega_{k} t} \hat{a}_{k}^{\dagger}-\hat{L}^{\dagger} g_{k} e^{-i \Omega_{k} t} \hat{a}_{k}\right],
$$

where $\Omega_{k}=\omega_{k}-\Omega$. Here we have finally restricted the form of $\hat{H}_{\Omega}$ to be such that $\hat{L}$ in the interaction picture simply rotates in the complex plane at frequency $\Omega$. That is $\hat{L}_{\text {int }}(t)=\hat{L} e^{-i \Omega t}$.

\section{NON-MARKOVIAN STOCHASTIC SCHRÖDINGER EQUATIONS DERIVED}

In this section we show that diffusive non-Markovian SSEs have an interpretation under the modal interpretation of quantum mechanics. To do this we choose a decomposition such that the preferred projectors have the form

$$
\hat{\pi}_{\left\{q_{k}\right\}}=\left|\left\{q_{k}\right\}\right\rangle\left\langle\left.\left\{q_{k}\right\}\right|_{\text {bath }} \otimes \hat{1}_{\text {sys }} .\right.
$$

This means the bath is given definite properties, while the system is treated as a purely quantum system, which nevertheless influences the bath values via the coupling Hamiltonian. The different unravelings correspond to different choices of $\left|\left\{q_{k}\right\}\right\rangle\left\langle\left.\left\{q_{k}\right\}\right|_{\text {bath }}\right.$. For the overcomplete unravelings, like the coherent state unraveling $[5,10]$ we have to use a POM [32]. This means we have to use the enlarged Hilbert space preferred projector $\hat{\Pi}_{\left\{q_{k}\right\}}$, which in general form is

$$
\hat{\Pi}_{\left\{q_{k}\right\}}=\left|\left\{q_{k}\right\}\right\rangle\left\langle\left.\left\{q_{k}\right\}\right|_{\text {bath }+ \text { aux }} \otimes \hat{1}_{\text {sys }} .\right.
$$

With these projectors, the property states $\left|\Psi_{n}(t)\right\rangle$ [similarly for $\left.\left|\Phi_{n}(t)\right\rangle\right]$ can be factorized as

$$
\left|\Psi_{n}(t)\right\rangle=\left|\left\{q_{k}\right\}\right\rangle\left|\psi_{\left\{q_{k}\right\}}(t)\right\rangle,
$$

where $\left|\psi_{\left\{q_{k}\right\}}(t)\right\rangle$ is called the conditioned system state. It receives this name because it lives entirely in $\mathcal{H}_{\text {sys }}$ and is conditioned on the bath values $\left\{q_{k}\right\}$. The form of $\left|\psi_{\left\{q_{k}\right\}}(t)\right\rangle$ is

$$
\left|\psi_{\left\{q_{k}\right\}}(t)\right\rangle=\left\langle\left\{q_{k}\right\} \mid \Psi(t)\right\rangle / \sqrt{N},
$$

where the normalization constant is defined as

$$
N=\left\langle\Psi(t) \mid\left\{q_{k}\right\}\right\rangle\left\langle\left\{q_{k}\right\} \mid \Psi(t)\right\rangle .
$$

This is the state of the system conditioned on the bath hidden variables having values $\left\{q_{k}\right\}$.

For an actual trajectory (in the sense of Ref. [10]), the bath values $\left\{q_{k}(t)\right\}$ are time dependent. This state becomes $\left|\psi_{\left\{q_{k}(t)\right\}}(t)\right\rangle$ and represents the state of the system conditioned on the bath having this trajectory. That is, it is continuous in time and the differential equation of this state will represent its evolution. In Refs. [10] and [11] we showed that by starting with Eq. (4.4), the time derivative of this equation gives diffusive non-Markovian SSEs. Thus in this paper we will not reproduce these derivations, but instead show that by using our velocity operator technique we can rederive the actual trajectories for $\left\{q_{k}(t)\right\}$. This shows that diffusive non-Markovian SSEs have a modal interpretation. If fact, because the orthodox interpretation only gives an interpretation for the solutions of non-Markovian SSE at a particular time (time of measurement), we believe that the only nontrivial interpretation of non-Markovian SSEs is a modal interpretation. 
Before we consider specific unravelings we would like to note that the velocity field, Eq. (2.25), can be written in terms of the conditioned system state as

$$
v_{k}\left(\left\{q_{k}\right\}, t\right)=\operatorname{Re}\left[\left\langle\psi_{\left\{q_{k}\right\}}(t)\left|\overrightarrow{\hat{v}_{k}\left(\left\{q_{k}\right\}, t\right)}\right| \psi_{\left\{q_{k}\right\}}(t)\right\rangle\right],
$$

where

$$
\overrightarrow{\hat{v}_{k}\left(\left\{q_{k}\right\}, t\right)}\left|\psi_{\left\{q_{k}\right\}}(t)\right\rangle \equiv\left\langle\left\{q_{k}\right\}\left|\hat{v}_{k}(t)\right| \Psi(t)\right\rangle / \sqrt{N} .
$$

This results in the following differential equation for the bath values:

$$
d_{t} q_{k}(t)=\operatorname{Re}\left[\left\langle\psi_{\left\{q_{k}(t)\right\}}(t)\left|\overrightarrow{\hat{v}_{k}\left(\left\{q_{k}(t)\right\}, t\right)}\right| \psi_{\left\{q_{k}(t)\right\}}(t)\right\rangle\right],
$$

where $\left|\psi_{\left\{q_{k}(t)\right\}}(t)\right\rangle=\left.\left|\psi_{\left\{q_{k}\right\}}(t)\right\rangle\right|_{\left\{q_{k}=q_{k}(t)\right\}}$.

\section{A. Position unraveling}

The first unraveling we consider is the position unraveling. This results when we chose a preferred projective measure of the form

$$
\left\{\hat{\pi}_{\left\{q_{k}\right\}}=\hat{\pi}_{\left\{x_{k}\right\}}=\left|\left\{x_{k}\right\}\right\rangle\left\langle\left.\left\{x_{k}\right\}\right|_{\text {bath }} \otimes \hat{1}_{\text {sys }}\right\},\right.
$$

where $\left|\left\{x_{k}\right\}\right\rangle$ is the multimode eigenstate of the position operators $\hat{x}_{k}=\left(\hat{a}_{k}^{\dagger}+\hat{a}_{k}\right) / \sqrt{2}$.

To simplify the overall equation we define a noise function $z(t)$ as

$$
z(t)=\sum_{k} g_{k} \sqrt{2} x_{k}(t) e^{-i \Omega_{k} t}
$$

In Ref. [11] we showed that the non-Markovian SSE for the position unraveling is

$$
\begin{aligned}
d_{t}\left|\psi_{z}(t)\right\rangle= & \left\{-\frac{i}{\hbar} \hat{H}_{\mathrm{int}}(t)+\left(\hat{L}-\langle\hat{L}\rangle_{t}\right) z^{*}(t)-(\hat{L}\right. \\
& \left.-\langle\hat{L}\rangle_{t}\right)\left[\hat{B}_{z}(t)+\hat{D}_{z}(t)\right]+\left\langle( \hat { L } - \langle \hat { L } \rangle _ { t } ) \left[\hat{B}_{z}(t)\right.\right. \\
& \left.\left.+\hat{D}_{z}(t)\right]\right\rangle_{t}-\left(\hat{L}^{\dagger}-\left\langle\hat{L}^{\dagger}\right\rangle_{t}\right)\left[\hat{A}_{z}(t)+\hat{C}_{z}(t)\right] \\
& +\left\langle\left(\hat{L}^{\dagger}-\left\langle\hat{L}^{\dagger}\right\rangle_{t}\right)\left[\hat{A}_{z}(t)+\hat{C}_{z}(t)\right]\right\rangle_{t} \\
& \times\}\left|\psi_{z}(t)\right\rangle,
\end{aligned}
$$

where $\left|\psi_{z}(t)\right\rangle \equiv\left|\psi_{\left\{x_{k}(t)\right\}}(t)\right\rangle$ and $\langle\hat{L}\rangle_{t}=\left\langle\psi_{z}(t)|\hat{L}| \psi_{z}(t)\right\rangle$. The four operators $\hat{A}_{z}(t), \hat{B}_{z}(t), \hat{C}_{z}(t)$ and $\hat{D}_{z}(t)$ are defined as ansatzen to functional derivatives. It turns out that in general these operators are not solvable. The perturbation techniques outlined in Refs. [15] and [16] can be applied to this non-Markovian SSE to give a perturbative solution. Given that there is no Markovian limit to this equation, however, it is unclear whether such perturbative methods would be effective.

We also showed, after considerable effort, that

$$
d_{t} x_{k}(t)=\left[\langle\hat{L}\rangle_{t} g_{k} e^{i \Omega_{k} t}+\left\langle\hat{L}^{\dagger}\right\rangle_{t} g_{k} e^{-i \Omega_{k} t}\right] / \sqrt{2} .
$$

Integrating this gives

$$
x_{k}(t)=x_{k}(0)+\int_{0}^{t} d s\left[\langle\hat{L}\rangle_{s} g_{k} e^{i \Omega_{k} s}+\left\langle\hat{L}^{\dagger}\right\rangle_{s} g_{k} e^{-i \Omega_{k} s}\right] / \sqrt{2}
$$

where $x_{k}(0)$ is the random variable chosen from the distribution

$$
P\left(\left\{x_{k}\right\}, 0\right)=\left|\left\langle\left\{x_{k}\right\} \mid\left\{0_{k}\right\}\right\rangle\right|^{2}=\prod_{k} \frac{\exp \left(-x_{k}^{2}\right)}{\sqrt{\pi}} .
$$

That is, we have chosen the initial condition $|\Psi(t)\rangle=$ $\left|\left\{0_{k}\right\}\right\rangle|\psi(0)\rangle$.

To show that Eq. (4.8) does give the same trajectories for the values $\left\{x_{k}(t)\right\}$ as in Eq. (4.13), we apply the Hamiltonians defined in Sec. III to Eq. (2.26), with $q_{k}=x_{k}$. This gives

$$
\hat{v}_{k}(t)=\left[g_{k} e^{i \Omega_{k} t} \hat{L}+g_{k} e^{-i \Omega_{k} t} \hat{L}^{\dagger}\right] / \sqrt{2},
$$

as $\left[\hat{x}_{k}, \hat{a}_{k}\right]=-1 / \sqrt{2}$ and $\left[\hat{x}_{k}, \hat{a}_{k}^{\dagger}\right]=1 / \sqrt{2}$. Substituting this into Eq. (4.6) gives a velocity field of the form

$$
\begin{aligned}
v_{k}\left(\left\{x_{k}\right\}, t\right)= & {\left[g_{k} e^{i \Omega_{k} t}\left\langle\psi_{\left\{x_{k}\right\}}(t)|\hat{L}| \psi_{\left\{x_{k}\right\}}(t)\right\rangle+g_{k} e^{-i \Omega_{k} t}\right.} \\
& \left.\times\left\langle\psi_{\left\{x_{k}\right\}}(t)\left|\hat{L}^{\dagger}\right| \psi_{\left\{x_{k}\right\}}(t)\right\rangle\right] / \sqrt{2} .
\end{aligned}
$$

Thus Eq. (4.8) immediately reproduces Eq. (4.12), thereby confirming that the modal theory does give the same non-Markovian SSEs, as found with the orthodox theory.

\section{B. Quadrature unraveling}

The next unraveling we consider is what we call the quadrature unraveling. In Ref. [10] we show that this unraveling only exists for certain environments, such that for every mode $k$ in the bath there exists another mode, which we can label $-k$, such that $\Omega_{-k}=-\Omega_{k}$ and $g_{-k}=g_{k}$. These simply imply that the modes coupled to the system come in symmetric pairs about the system frequency $\Omega$. The form of the preferred projective measure for this unraveling is

$\left\{\hat{\pi}_{\left\{q_{k}\right\}}=\hat{\pi}_{\left\{X_{k}^{+}, Y_{k}^{-}\right\}}=\left|\left\{X_{k}^{+}, Y_{k}^{-}\right\}\right\rangle\left\langle\left.\left\{X_{k}^{+}, Y_{k}^{-}\right\}\right|_{\text {bath }} \otimes \hat{1}_{\text {sys }}\right\}\right.$,

where $\left|\left\{X_{k}^{+}, Y_{k}^{-}\right\}\right\rangle=\prod_{k>0}\left|X_{k}^{+}, Y_{k}^{-}\right\rangle$where $\left|X_{k}^{+}, Y_{k}^{-}\right\rangle$is the two mode entangled (EPR) state

$$
\left|X_{k}^{+}, Y_{k}^{-}\right\rangle=\int \frac{d x^{\prime}}{\sqrt{2 \pi}}\left|\frac{X_{k}^{+}-x^{\prime}}{\sqrt{2}}\right\rangle_{-k}\left|\frac{X_{k}^{+}+x^{\prime}}{\sqrt{2}}\right\rangle_{k} e^{i Y_{k}^{-} x^{\prime}} .
$$

Here $\left|\left(X_{k}^{+}+x^{\prime}\right) / \sqrt{2}\right\rangle_{k}$ is an eigenstate of $\hat{x}_{k}$, and similarly $\left|\left(X_{k}^{+}-x^{\prime}\right) / \sqrt{2}\right\rangle_{-k}$ for $\hat{x}_{-k}$. Equation (4.18) is an eigenstate of both the operators

$$
\begin{aligned}
& \hat{X}_{k}^{+}=\left(\hat{x}_{k}+\hat{x}_{-k}\right) / \sqrt{2}, \\
& \hat{Y}_{k}^{-}=\left(\hat{y}_{k}-\hat{y}_{-k}\right) / \sqrt{2},
\end{aligned}
$$


where $\hat{x}_{k}$ and $\hat{y}_{k}$ are the quadratures of $\hat{a}_{k}$ :

$$
\hat{a}_{k}=\left(\hat{x}_{k}+i \hat{y}_{k}\right) / \sqrt{2} .
$$

As in the position unraveling we define a noise function $z(t)$ as

$$
z(t)=\sum_{k>0} 2 g_{k}\left[X_{k}^{+}(t) \cos \left(\Omega_{k} t\right)+Y_{k}^{-}(t) \sin \left(\Omega_{k} t\right)\right],
$$

which by definition is real. In Ref. [10] we showed that the non-Markovian SSE for the quadrature unraveling is

$$
\begin{aligned}
d_{t}\left|\psi_{z}(t)\right\rangle= & {\left[-\frac{i}{\hbar} \hat{H}_{\mathrm{int}}(t)-\left(\hat{L}_{x}-\left\langle\hat{L}_{x}\right\rangle_{t}\right) \hat{Q}_{z}(t)\right.} \\
& +\left\langle\left(\hat{L}_{x}-\left\langle\hat{L}_{x}\right\rangle_{t}\right) \hat{Q}_{z}(t)\right\rangle_{t} \\
& \left.+z(t)\left(\hat{L}-\langle\hat{L}\rangle_{t}\right)\right]\left|\psi_{z}(t)\right\rangle
\end{aligned}
$$

where $\left|\psi_{z}(t)\right\rangle \equiv\left|\psi_{\left\{X_{k}^{+}(t), Y_{k}^{-}(t)\right\}}(t)\right\rangle$ and $\hat{L}_{x}=\hat{L}+\hat{L}^{\dagger}$. Again the operator $\hat{Q}_{z}(t)$ is an ansatz to a functional derivative. In Ref. [16] we outlined a perturbation technique for finding this operator, if an exact solution cannot be found.

The differential equations for $X_{k}^{+}(t)$ and $Y_{k}^{-}(t)$ were shown, using the method of characteristics, to be

$$
\begin{aligned}
d_{t} X_{k}^{+}(t) & =g_{k} \cos \left(\Omega_{k} t\right)\left\langle\hat{L}_{x}\right\rangle_{t} \\
d_{t} Y_{k}^{-}(t) & =g_{k} \sin \left(\Omega_{k} t\right)\left\langle\hat{L}_{x}\right\rangle_{t} .
\end{aligned}
$$

Integrating these differential equation from time 0 to $t$ we get

$$
\begin{aligned}
& X_{k}^{+}(t)=X_{k}^{+}(0)+\int_{0}^{t} g_{k} \cos \left(\Omega_{k} s\right)\left\langle\hat{L}_{x}\right\rangle_{s} d s, \\
& Y_{k}^{-}(t)=Y_{k}^{-}(0)+\int_{0}^{t} g_{k} \sin \left(\Omega_{k} s\right)\left\langle\hat{L}_{x}^{\dagger}\right\rangle_{s} d s .
\end{aligned}
$$

As in the position unraveling the random variables $X_{k}^{+}(0)$ and $Y_{k}^{-}(0)$ are chosen from the initial distribution. For this unraveling and the initial condition $|\Psi(t)\rangle=\left|\left\{0_{k}\right\}\right\rangle|\psi(0)\rangle$ this distribution is

$$
P\left(\left\{X_{k}^{+}, Y_{k}^{-}\right\}, 0\right)=\prod_{k>0} \frac{e^{-\left(X_{k}^{+2}+Y_{k}^{-2}\right)}}{\pi} .
$$

To show that Eqs. (4.24) and (4.25) can be derived from the modal theory (velocity operator technique) we apply the Hamiltonians defined in Sec. III to Eq. (2.26). For this unraveling the set of velocity operators $\left\{\hat{v}_{k}\right\}$ will be the union of $\left\{\hat{v}_{k}^{+}\right\}$and $\left\{\hat{v}_{k}^{-}\right\}$, where

$$
\begin{aligned}
& \hat{v}_{k}^{+}(t)=\frac{-i}{\hbar}\left[\hat{X}_{k}^{+}, \hat{H}_{\mathrm{uni}}\right]=g_{k} \hat{L}_{x} \cos \left(\Omega_{k} t\right), \\
& \hat{v}_{k}^{-}(t)=\frac{-i}{\hbar}\left[\hat{Y}_{k}^{-}, \hat{H}_{\mathrm{uni}}\right]=g_{k} \hat{L}_{x} \sin \left(\Omega_{k} t\right),
\end{aligned}
$$

which are both real by definition. Substituting these velocity operators into Eq. (4.6) gives

$$
\begin{aligned}
v_{k}^{+}\left(\left\{X_{k}^{+}, Y_{k}^{-}\right\}, t\right)= & g_{k}\left\langle\psi_{\left\{X_{k}^{+}, Y_{k}^{-}\right\}}(t)\left|\hat{L}_{x}\right| \psi_{\left\{X_{k}^{+}, Y_{k}^{-}\right\}}(t)\right\rangle \\
& \times \cos \left(\Omega_{k} t\right), \\
v_{k}^{+}\left(\left\{X_{k}^{+}, Y_{k}^{-}\right\}, t\right)= & g_{k}\left\langle\psi_{\left\{X_{k}^{+}, Y_{k}^{-}\right\}}(t)\left|\hat{L}_{x}\right| \psi_{\left\{X_{k}^{+}, Y_{k}^{-}\right\}}(t)\right\rangle \\
& \times \sin \left(\Omega_{k} t\right) .
\end{aligned}
$$

Thus Eq. (4.8) simply yields Eqs. (4.24) and (4.25). Thus the modal theory gives the correct non-Markovian SSE.

\section{Coherent unraveling}

The last unraveling we consider is the coherent state unraveling. This non-Markovian SSE was first presented by Diósi, Gisin, and Strunz [5]. In Ref. [10] we showed that it could be derived in the orthodox interpretation by considering a bath measurement in terms of the Husimi POM [35]. This has POM elements

$$
\hat{F}_{\left\{a_{k}\right\}}=\frac{1}{\pi^{K}}\left|\left\{a_{k}\right\}\right\rangle\left\langle\left\{a_{k}\right\}\right|,
$$

where $\hat{a}_{k}\left|a_{k}\right\rangle=a_{k}\left|a_{k}\right\rangle$ and $K$ is the total number of modes. The noise function $z(t)$ for this unraveling is

$$
z(t)=\sum_{k} g_{k} a_{k}(t) e^{-i \Omega_{k} t} .
$$

In Refs. $[5,10]$ it is shown that the non-Markovian SSE for the coherent unraveling is

$$
\begin{aligned}
d_{t}\left|\psi_{z}(t)\right\rangle= & {\left[-\frac{i}{\hbar} \hat{H}_{\mathrm{int}}(t)-\left(\hat{L}^{\dagger}-\left\langle\hat{L}^{\dagger}\right\rangle_{t}\right) \hat{C}_{z}(t)\right.} \\
& +\left\langle\left(\hat{L}^{\dagger}-\left\langle\hat{L}^{\dagger}\right\rangle_{t}\right) \hat{C}_{z}(t)\right\rangle_{t} \\
& \left.+z^{*}(t)\left(\hat{L}-\langle\hat{L}\rangle_{t}\right)\right]\left|\psi_{z}(t)\right\rangle,
\end{aligned}
$$

where $\left|\psi_{z}(t)\right\rangle \equiv\left|\psi_{\left\{a_{k}(t)\right\}}(t)\right\rangle$. The operator $\hat{C}_{z}(t)$ as in the previous unravelings is an ansatz to a functional derivative. As in the quadrature unraveling perturbation techniques exist for finding a perturbative solution for this ansatz $[15,16]$.

Using the same procedure as the other unravelings, the differential equation for $a_{k}(t)$ is

$$
d_{t} a_{k}^{*}(t)=g_{k} e^{-i \Omega_{k} t}\left\langle\hat{L}^{\dagger}\right\rangle_{t},
$$

which integrates to give

$$
a_{k}^{*}(t)=a_{k}^{*}(0)+\int_{0}^{t} g_{k} e^{-i \Omega_{k} s}\left\langle\hat{L}^{\dagger}\right\rangle_{s} d s .
$$

For an initial vacuum bath state, the random variable $a_{k}^{*}(0)$ is defined by the initial distribution

$$
P\left(\left\{a_{k}\right\}, 0\right)=\prod_{k} \frac{e^{-\left|a_{k}\right|^{2}}}{\pi} .
$$


To show that the modal theory can be used to describe this non-Markovian SSE we have to find the projector in $\mathcal{K}$ which is equivalent to the POM elements defined in Eq. (4.33). In Ref. [32] we showed that for a single mode this projector is $\left|x^{+}, y^{-}\right\rangle\left\langle x^{+}, y^{-}\right|$where

$$
\left|x^{+}, y^{-}\right\rangle=\int \frac{d x^{\prime}}{\sqrt{2 \pi}}\left|\frac{x^{+}-x^{\prime}}{\sqrt{2}}\right\rangle_{\text {aux }}\left|\frac{x^{+}+x^{\prime}}{\sqrt{2}}\right\rangle_{\text {uni }} e^{i y^{-} x^{\prime}},
$$

where the states in the integrand are $x$ states. Thus the multimode projector used to define this unraveling is

$$
\hat{\Pi}_{\left\{q_{k}\right\}}=\hat{\Pi}_{\left\{a_{k}\right\}}=\left|\left\{x_{k}^{+}, y_{k}^{-}\right\}\right\rangle\left\langle\left.\left\{x_{k}^{+}, y_{k}^{-}\right\}\right|_{\text {bath }+ \text { aux }} \otimes \hat{1}_{\text {sys }},\right.
$$

where $a_{k}$ is defined by

$$
a_{k}=x_{k}^{+}+i y_{k}^{-} .
$$

This allows us to define the operator $\hat{A}_{k}$, such that

$$
\hat{A}_{k}\left|x^{+}, y^{-}\right\rangle=a_{k}\left|x^{+}, y^{-}\right\rangle
$$

as

$$
\hat{A}_{k}=\hat{x}_{k}^{+}+i \hat{y}_{k}^{-}=\hat{a}_{k}+\hat{b}_{k}^{\dagger},
$$

which is a normal operator [36]. Here $\hat{x}_{k}^{+}$and $\hat{y}_{k}^{-}$are defined as

$$
\begin{aligned}
& \hat{x}_{k}^{+}=\left[\hat{a}_{k}+\hat{a}_{k}^{\dagger}+\hat{b}_{k}+\hat{b}_{k}^{\dagger}\right] / 2, \\
& \hat{y}_{k}^{-}=\left[-i \hat{a}_{k}+i \hat{a}_{k}^{\dagger}+i \hat{b}_{k}-i \hat{b}_{k}^{\dagger}\right] / 2,
\end{aligned}
$$

where $\hat{b}_{k}$ and $\hat{b}_{k}^{\dagger}$ are annihilation and creation operators which act in $\mathcal{H}_{\text {aux }}$. In this enlarged Hilbert space the velocity operators are

$$
\begin{aligned}
\hat{v}_{k}^{+}(t) & =-\frac{i}{\hbar}\left[\hat{x}_{k}^{+}, \hat{H}_{\mathrm{uni}} \otimes \hat{1}_{\mathrm{aux}}\right] \\
& =\left[g_{k} e^{i \Omega_{k} t} \hat{L}+g_{k} e^{-i \Omega_{k} t} \hat{L}^{\dagger}\right] / 2, \\
\hat{v}_{k}^{-}(t) & =-\frac{i}{\hbar}\left[\hat{y}_{k}^{-}, \hat{H}_{\mathrm{uni}} \otimes \hat{1}_{\mathrm{aux}}\right] \\
& =\left[-i g_{k} e^{i \Omega_{k} t} \hat{L}+i g_{k} e^{-i \Omega_{k} t} \hat{L}^{\dagger}\right] / 2 .
\end{aligned}
$$

With these velocity operators the velocity fields become

$$
\begin{aligned}
v_{k}^{+}\left(\left\{x_{k}^{+}, y_{k}^{-}\right\}, t\right)= & \left\langle\psi_{\left\{x_{k}^{+}, y_{k}^{-}\right\}}(t)\right|\left[g_{k} e^{i \Omega_{k} t} \hat{L}+g_{k} e^{-i \Omega_{k} t}\right. \\
& \left.\times \hat{L}^{\dagger}\right]\left|\psi_{\left\{x_{k}^{+}, y_{k}^{-}\right\}}(t)\right\rangle / 2 \\
v_{k}^{-}\left(\left\{x_{k}^{+}, y_{k}^{-}\right\}, t\right)= & \left\langle\psi_{\left\{x_{k}^{+}, y_{k}^{-}\right\}}(t)\right|\left[-i g_{k} e^{i \Omega_{k} t} \hat{L}+i g_{k}\right. \\
& \left.\times e^{-i \Omega_{k} t} \hat{L}^{\dagger}\right]\left|\psi_{\left\{x_{k}^{+}, y_{k}^{-}\right\}}(t)\right\rangle / 2
\end{aligned}
$$

Substituting these into Eq. (4.8) gives

$$
\begin{aligned}
& d_{t} x_{k}^{+}(t)=\left[\langle\hat{L}\rangle_{t} g_{k} e^{i \Omega_{k} t}+\left\langle\hat{L}^{\dagger}\right\rangle_{t} g_{k} e^{-i \Omega_{k} t}\right] / 2, \\
& d_{t} y_{k}^{-}(t)=\left[-i\langle\hat{L}\rangle_{t} g_{k} e^{i \Omega_{k} t}+i\left\langle\hat{L}^{\dagger}\right\rangle_{t} g_{k} e^{-i \Omega_{k} t}\right] / 2 .
\end{aligned}
$$

Since $a_{k}=x_{k}^{+}+i y_{k}^{-}$we once again easily obtain Eq. (4.36) as found from the orthodox theory.

\section{DISCUSSION AND CONCLUSIONS}

Given the success of continuous quantum measurement theory [23] in giving a nontrivial interpretation of Markovian SSEs, it is natural to seek a similar interpretation for diffusive non-Markovian SSEs. It turns out that to give a meaning to such non-Markovian SSEs we have to consider the modal interpretation of quantum mechanics. This is because under the orthodox interpretation of quantum mechanics, only the solution of the non-Markovian SSE at a time $t$ can be given a meaning [10]. It corresponds to the state of the system at that time given a measurement on the bath at that time yielding a particular result. The bath cannot be measured continuously because non-Markovian systems have a memory, so past measurements of the bath will in general have a back-action which disrupts the system's average evolution. Thus the solution at a particular time may have an interpretation, but the linking of these solutions at different times does not. The trajectory generated by the non-Markovian SSE can be regarded only as a numerical tool for calculating a conditioned state at a particular time.

However, under the modal interpretation, in particular a view which is closest in line with Bell's beable theory $[26,30]$, we find that non-Markovian SSEs do have a nontrivial interpretation. In this interpretation, the bath has definite properties even if it is not measured, so the backaction problem disappears. The system is treated as a purely quantum system which, however, depends upon the values of the bath properties: the values of the bath hidden variables. The evolution of the system is generated by the non-Markovian SSE and the system state guides the values of the hidden variables for the bath. The bath hidden variables are similar to Bohmian hidden variables [33], as they obey a deterministic differential equation with stochastic initial conditions. In fact, one unraveling we have considered corresponds to Bohmian mechanics of the bath in an interaction frame.

In this paper we also considered the quadrature and coherent unravelings [10]. The different unravelings are determined by choosing which bath observable is to be given property status. The noise $z(t)$ which appears in the non-Markovian SSE is simply a linear combination of the time-dependent values of the bath hidden variables. The quadrature unraveling is defined such that $z(t)$ is real, while for the coherent unraveling it is complex. In the Markovian limit the former becomes the quantum trajectory for homodyne detection and the latter becomes the quantum trajectory for heterodyne detection. Thus quantum trajectories have both the standard continuous measurement interpretation and the above modal interpretation.

In conclusion it seems that to give diffusive nonMarkovian SSE a satisfying interpretation we must give up the orthodox interpretation of quantum mechanics and consider the lesser known but equally valid modal interpretation. In Refs. [37, 38] it is claimed that the formalism presented there does give a satisfying interpreta- 
tion of non-Markovian SSEs. If this is correct, we must conclude that said formalism is a hidden-variable theory. The clarification of this issue is beyond the scope of the current paper. Another problem for future work would be to determine what choices for objective properties of the bath will give rise to SSEs with a Markovian limit. Finally, it would be interesting to use the modal theory to develop discontinuous non-Markovian SSEs, such as those which corresponds to a number state decomposi- tion.

\section{Acknowledgments}

This work was supported by the Australian Research Council.
[1] H.J. Carmichael, An Open Systems Approach to Quantum Optics (Springer-Verlag, Berlin, 1993).

[2] S. Nakajima, Prog. Theor. Phys. 20, 948 (1958).

[3] R. Zwanzig, J. Chem. Phys. 33, 1338 (1960).

[4] L. Diósi and W.T. Strunz, Phys. Lett. A 235, 569 (1997).

[5] L. Diósi, N. Gisin, and W.T. Strunz, Phys. Rev. A 58, 1699 (1998).

[6] W.T. Strunz, L. Diósi and N. Gisin, Phys. Rev. Lett. 82, 1801 (1999).

[7] P. Gaspard and M. Nagaoka, J. Chem. Phys 111, 5676 (1999).

[8] J.D. Cresser, Laser Phys. 10, 1 (2000).

[9] A.A. Budini, Phys. Rev. A 63, 012106 (2000).

[10] J. Gambetta and H.M. Wiseman, Phys. Rev. A 66, 012108 (2002).

[11] J. Gambetta and H.M. Wiseman, Fluctuations and Noise in Photonics and Quantum Optics, edited by D. Abbott, J. H. Shapiro, and Y. Yamamoto, Proceedings of SPIE, Vol. 5111(SPIE, Bellingham, WA, 2003), pp 313-324.

[12] A. Bassi and G. Ghirardi, Phys. Rev. A 65, 042114 (2002).

[13] A. Bassi, e-print quant-ph 0209170 (2002).

[14] M. W. Jack and M. J. Collett, Phys. Rev. A 61, 062106 (2000).

[15] T. Yu, L. Diósi, N. Gisin, and W.T. Strunz, Phys. Rev. A 60, 91 (1999).

[16] J. Gambetta and H.M. Wiseman, Phys. Rev. A 66, 052105 (2002).

[17] J. Dalibard, Y. Castin, and K. Mølmer, Phys. Rev. Lett. 68, 580 (1992).

[18] N. Gisin, Helv. Phys. Acta. 62, 363 (1989).

[19] L. Diósi, J. Phys. A 21, 2885 (1989).

[20] P. Pearle, Phys. Rev. A 39, 2277 (1989).

[21] H. M. Wiseman and G. J. Milburn, Phys. Rev. A 47, 642 (1993).

[22] H. M. Wiseman and G. J. Milburn, Phys. Rev. A. 47,
1652 (1993).

[23] H.M. Wiseman, Quantum Semiclassic. Opt. 8, 205 (1996).

[24] K. Kraus, States, Effects, and Operations: Fundamental Notions of Quantum Theory (Springer, Berlin, 1983).

[25] W.T. Strunz (personal communication) has also sought an interpretation of non-Markovain SSEs, but not along the lines explored in this paper.

[26] J. S. Bell, CERN-TH.4035/84 (1984). Reprinted in John S. Bell on the Foundations of Quantum Mechanics, edited by M. Bell, K. Gottfried and M. Veltman (World Scientific, Singapore, 2001).

[27] J. Bub, Interpretating the Quantum World (Cambridge University Press, Cambridge, 1997).

[28] P. E. Vermass and D. Dieks, Found. Phys. 25, 145 (1995).

[29] G. Bacciagaluppi and M. Dickson, Found. Phys. 29, 1165 (1999).

[30] A. Sudbery, Stud. Hist. Phil. Mod. Phys. 33, 387 (2002).

[31] R. W. Spekkens and J. E. Sipe, Found. Phys. 31, 1431 (2001).

[32] J. Gambetta and H.M. Wiseman, to be published in Found. Phys. (2003). e-print quant-ph/0306145

[33] D. Bohm, Phys. Rev. 85, 166 (1952); 85, 180 (1952).

[34] C. W. Helstrom, Quantum Detection and Estimation Theory (Academic Press, New York, 1976).

[35] K. Husimi, Proc. Phys. Math. Soc. Jpn. 22, 264 (1940); D. F. Walls and G. J. Milburn, Quantum Optics (Springer, Berlin, 1994).

[36] M. A. Nielsen and I. L. Chuang, Quantum Computation and Quantum Information (Cambridge University Press, New York, 2000).

[37] E. R. Loubenets, J. Phys. A 34, 7639 (2001).

[38] O.E. Barndorff-Nielsen and E. R. Loubenets, J. Phys. A 35, 565 (2002). 